\title{
Editorial
}

\section{Pulse pressure and prognosis}

Many epidemiological studies have demonstrated the close relation between blood pressure and the subsequent development of cardiovascular disease. Blood pressure has characteristically been defined by its direct relation with cardiac output and peripheral resistance. In essential hypertension, the most consistent cause for an elevated blood pressure is an increase in peripheral resistance, rather than cardiac output. This increase in peripheral resistance, considered to be caused by arteriolar constriction, was shown in early physiologic experiments to be best represented by an increase in diastolic pressure. ${ }^{1}$ Consequently, it was generally believed that the hazard of hypertension to the cardiovascular system was derived principally from the diastolic component of blood pressure. This viewpoint was subsequently reinforced by a number of therapeutic trials, showing the benefits of treating hypertension defined on the basis of elevated diastolic blood pressures. At the same time, systolic blood pressure was considered to reflect elasticity of the large arteries and any increases in systolic pressure were viewed as a natural and innocuous effect of increased stiffness of the aorta caused by aging. However, in recent years, the emphasis on increased peripheral resistance and elevated diastolic pressure as the principal determinants of cardiovascular outcome has been challenged. This is in part due to a reappraisal of the early studies and the emergence of new insights into the pathophysiological significance of increased arterial stiffness and its influence on blood pressure components, particularly systolic and pulse pressure.

As early as 1971, the Framingham study showed that although diastolic pressure was the major determinant of cardiovascular risk in men under 45 years of age, systolic blood pressure was the stronger risk factor in older men and in women of all ages. ${ }^{2}$ Since then, a meta-analysis of cohort studies $^{3}$ has confirmed a continuous, graded, and independent association between systolic blood pressure and the risk of coronary heart disease, stroke, and end stage renal disease which is stronger than that for diastolic blood pressure and is most evident in men. Moreover, several large scale prospective studies have reported up to a threefold increase in stroke and up to a 2.2 fold increase in myocardial infarction among those with isolated systolic hypertension. ${ }^{4}$ The latter studies provided the first indirect evidence that a widened pulse pressure was an indicator of cardiovascular risk, but only recently has pulse pressure per se been evaluated.

\section{Pulse pressure in normotensive and hypertensive populations}

A recently published 20 year follow up study of almost 20000 normotensive and hypertensive subjects aged 40-69 years found pulse pressure to be an independent predictor of all cause, total cardiovascular, and especially coronary mortality in men of all age and mean blood pressure groups. ${ }^{5}$ In a series of 5730 untreated hypertensives followed for an average of five years, pulse pressure was found to be the only measure of blood pressure significantly and independently related to the in-treatment incidence of myocardial infarction. ${ }^{6}$ The use of ambulatory blood pressure measurement in hypertension also strongly supports the prognostic importance of pulse pressure. This effect seems to be most pronounced in older subjects? The Framingham data restricted to those over 50 years old showed that the association with coronary heart disease risk, although positive for systolic and diastolic blood pressure, was strongest for pulse pressure. ${ }^{8}$ Coronary risk increased with lower diastolic blood pressure at any level of systolic blood pressure greater than $120 \mathrm{~mm} \mathrm{Hg}$. A study conducted by the Veterans Administration ${ }^{9}$ showed that when systolic and diastolic blood pressure were considered jointly in a multivariate model containing clinical variables, systolic but not diastolic blood pressure was related to cardiovascular death in men aged $<60$ years. By contrast, in older men systolic blood pressure was positively and diastolic blood pressure negatively related to outcome, suggesting that pulse pressure was the best haemodynamic determinant of outcome in the elderly.

\section{Pulse pressure in ischaemic heart disease and left ventricular dysfunction}

Conventional wisdom might suggest that a reduced pulse pressure is associated with a worse outcome in patients with left ventricular dysfunction. However, the two studies that investigated the predictive value of pulse pressure in this patient population demonstrated the opposite effect. The SAVE (survival and ventricular enlargement) trial investigators found that a single sphygmomanometrically determined pulse pressure, measured 3-16 days following myocardial infarction in patients with asymptomatic left ventricular dysfunction, was positively associated with age, ejection fraction, female sex, hypertension, diabetes, and treatment with digoxin and calcium antagonists. ${ }^{10}$ In multivariate analysis, pulse pressure was an independent predictor of cardiovascular outcome, even after correction for mean arterial pressure. These findings were subsequently confirmed by the SOLVD (studies of left ventricular dysfunction) trial in patients with established asymptomatic and symptomatic heart failure. ${ }^{11}$ Moreover, in the latter trial mean arterial pressure was inversely related to total and cardiovascular mortality.

\section{Pathophysiological mechanisms}

Although the better correlation of systolic and pulse pressure with cardiovascular morbidity may in part reflect the fact that systolic pressure has an intrinsically wider range of values than diastolic and mean arterial pressure, important differences in the pathophysiological mechanisms that determine pulsatile and steady state pressure have been identified. Whereas mean arterial pressure is entirely determined by cardiac output and peripheral resistance governed by the arteriolar system, pulse pressure is influenced by the pattern of ventricular ejection, distensibility of the large arteries, the timing of reflected waves, and heart rate. ${ }^{12}$

The medial layer of the arterial wall consisting of smooth muscle cells, elastin, and collagen fibres is responsible for the viscoelastic properties of the large arteries. This enables the large arteries to regulate blood pressure by smoothing out the pulsations that occur with each ventricular ejection by instantaneously accommodating the volume of blood ejected 
from the heart, thereby storing part of the stroke volume during systolic ejection and preventing a precipitous increase in pressure. The intrinsic elastic recoil of the aorta during diastole preserves a steady flow of blood to the peripheral circulation, maintaining the level of diastolic blood pressure and allowing continuous perfusion of organs and tissues. However, the combined effects of intermittent stress and age result in fracture and fragmentation of elastin and a secondary increase in non-elastic collagen tissue. With the resultant increase in arterial stiffness the cushioning effect is lost and this leads to an increase in systolic pressure. In addition, the normal elastic recoil during diastole does not occur and the diastolic pressure tends to fall. Furthermore, based on pulsatile arterial haemodynamics, the blood pressure curve is due to the summation of the forward wave from the heart and a reflected wave from peripheral resistance vessels. Under normal conditions, the reflection waves occur during the diastolic component of the pressure wave, thereby maintaining diastolic and mean pressure (fig 1). However, with increased arterial stiffness pulse wave velocity increases greatly and additional reflecting points caused by atherosclerotic deposits may occur closer to the heart. This may cause a return of reflected waves during the systolic component of the pressure wave thereby further augmenting systolic and pulse pressure.

Given these physiological adaptations, the age related increase in systolic and pulse pressure and plateau or fall in diastolic blood pressure observed in cross sectional and longitudinal studies of elderly populations ${ }^{13}$ probably signifies the preponderance of increased arterial stiffness in this age group. Indeed, increased arterial stiffness is strongly influenced by aging, hypertension, and atheroma formation; an increased pulse pressure may be a composite manifestation of these processes.

The deleterious effect of systolic and pulse pressure may relate to the observation that biological materials are more susceptible to stress when it is intermittent rather than static and when it occurs at a faster rate. In addition, an
A
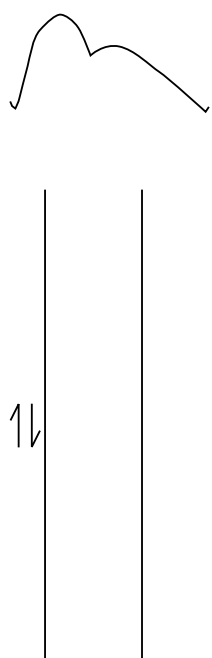

B
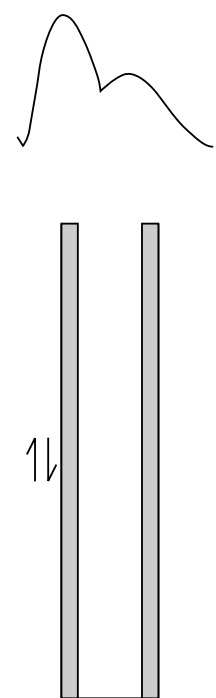
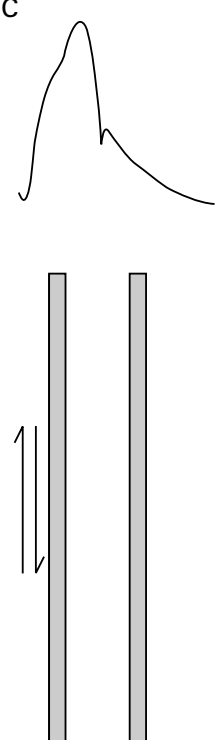

Figure 1 Relation between arterial compliance, pulse wave velocity, and the aortic pressure waveform. (A) Normal arterial compliance and normal pulse wave velocity leading to a secondary wave in diastole caused by wave reflection. (B) Increased arterial stiffness, but normal pulse wave velocity leading to increased amplitude of pressure waveform. (C) Increased arterial stiffness and increased pulse wave velocity leading to increased pressure wave amplitude and a late systolic peak caused by early wave reflection with absence of a secondary wave in diastole (isolated systolic hypertension). elevation in systolic blood pressure causes a disproportionate increase in cardiac workload, which in turn increases myocardial oxygen consumption, promotes left ventricular hypertrophy, and can compromise coronary perfusion. ${ }^{14}$ As perfusion to the coronary circulation occurs predominantly in diastole, a concomitant reduction in diastolic blood pressure may further impair myocardial blood flow and promote ischaemia, particularly in the presence of existing atherosclerotic disease.

\section{Clinical implications}

The association between risk and both systolic and pulse pressure creates an apparent anomaly for the clinician schooled in the importance of diastolic blood pressure. Although treatment guidelines for hypertension now emphasise the importance of systolic blood pressure control, in clinical practice systolic pressure does not appear to be as well controlled as diastolic pressure. ${ }^{15}$ It seems likely that lower diastolic pressures may still lull clinicians into a false sense of security when dealing with the elderly patient, whereas in fact cardiovascular risk may be increased in this situation. Unfortunately, however, there is little guidance on the role of pulse pressure in identifying the high risk candidate suitable for drug treatment. Moreover, few studies in hypertension and heart failure have evaluated the effect of drug treatment in specifically reducing pulse pressure and arterial stiffness. Preliminary data from experimental and clinical studies in hypertension suggest that while angiotensin converting enzyme inhibitors and calcium antagonists greatly improve arterial compliance, ${ }^{12}$ diuretics might lead to greater reductions in pulse pressure. ${ }^{16}$ Clearly, further information is required in the form of placebo controlled studies performing direct comparisons of the effect of the different classes of antihypertensive agents on arterial function, pulse pressure and subsequent cardiovascular morbidity and mortality. Nevertheless, despite caveats in current knowledge, the overwhelming message from the accumulating data is the need for greater attention to systolic and pulse pressure control, particularly in the elderly.

Waller Cardiac Department,

RAJDEEP S KHATTAR

St Mary's Hospital,

Praed Street,

London W2, UK

Cardiovascular Research Institute,

University of Leicester,

Leicester Royal Infirmary,

Leicester LE2 7LX, UK

1 Wiggers CJ. Physical and physiological aspects of arteriosclerosis and hypertension. Ann Intern Med 1932;6:12-30.

Kannel WB, Gordon T, Schwartz MJ. Systolic versus diastolic blood pressure and the risk of coronary heart disease. Am f Cardiol 1971;27:335-64.

$3 \mathrm{He}$ J, Whelton PK. Elevated systolic blood pressure as a risk factor for cardiovascular and renal disease. 7 Hypertension 1999;1 (suppl 2): S7-13.

Silagy CA McNeil JJ. Epidemiological aspects of isolated systolic hilagy CA, McNeil JJ. Epidemiological aspects of isolated systolic 1992;69:213-18.

5 Benetos A, Rudnichi A, Safar M, et al. Pulse pressure and cardiovascular mortality in normotensive and hypertensive subjects. Hypertension 1998;32: mortality

6 Fang J, Madhavan S, Cohen H, et al. Measures of blood pressure and myocardial infarction in treated hypertensive patients. F Hypertension 1995;13: 413-19.

7 Khattar RS, Swales JD, Dore C, et al. Prediction of coronary and cerebrovascular morbidity and mortality by direct continuous ambulatory blood pressure monitoring in essential hypertension. Circulation 1999;100:1071-6.

8 Franklin SS, Khan SA, Wong ND, et al. Is pulse pressure useful in predicting risk for coronary heart disease? The Framingham heart study. Circulation 1999;100:354-60.

9 Lee ML, Rosner BA, Weiss ST. Relationship of blood pressure to cardiovascular death: the effects of pulse pressure in the elderly. Ann Epidemiol 1999; 9:101-7.

10 Mitchell GF, Moye LA, Braunwald E, et al for the SAVE Investigators. Sphygmomanometrically determined pulse pressure is a powerful independent predictor of recurrent events after myocardial infarction in patients wendent predictor of recurrent events after myocardial infarction in pa

11 Domanski MJ, Mitchell GF, Norman JE, et al. Independent prognostic information provided by sphygmomanometrically determined pulse
ingentic information provided by sphygmomanometrically determined pulse
pressure and mean arterial pressure in patients with left ventricular dysfunction. F Am Coll Cardiol 1999;33;951-8. 
12 Safar M. Arteries in clinical hypertension. Philadelphia: Lippincott-Raven, 1994.

13 Whelton PK, He J, Klag MJ. Blood pressure in westernized populations. In: Swales JD, ed. Textbook of hypertension. London: Blackwell Scientific, 1994: 11-21.

14 Watanabe $\mathrm{H}$, Ohtsuka S, Kakihama $\mathrm{M}$, et al. Coronary circulation in dogs with experimental decrease in aortic compliance. I Am Coll Cardiol 1993;21:1497-506.
15 Swales JD. Current clinical practice in hypertension.: the EISBERG (evaluSwales JD. Current clinical practice in hypertension.: the EISBERG (evaluglobal) project. Am Heart f 1999; 138:S231-7.

16 Cushman WC, Materson BJ, Reda DJ, et al for the Veterans Affairs (VA) Cooperative Study Group on Antihypertensive Agents. Pulse pressure changes with six classes of antihypertensive agents and placebo [abstract]. Am $\mathcal{F}$ Hypertens 2000;13:22A.

\section{IMAGES IN CARDIOLOGY}

\section{A "new" coronary anomaly: origin of the right coronary artery below the aortic valve}

A 53 year old man presented with recurrent left thoracic pain suggesting coronary artery disease. Resting and exercise ECG showed no signs of ischaemia. Transthoracic echocardiography revealed all chambers to be of normal size and a normal left ventricular function. During coronary angiography, the ostium of the right coronary artery could not be found despite the use of various catheters. Injection of contrast medium into the left coronary artery showed a severely dilated proximal left anterior descending artery (LAD) (top right) with a huge tortuous collateral vessel to the right coronary artery (RCA) (bottom right). A retrograde flow was seen in the RCA. The LAD and the RCA distally to the collateral vessel were of normal diameter and there was no evidence of coronary artery disease.

Transoesophageal echocardiography confirmed the anomalous origin of the RCA from the left ventricular outflow tract (LVOT) below the aortic valve (AV) and the ascending aorta (AO) (below left). Colour Doppler flow studies revealed diastolic retrograde flow in the RCA into the LVOT (below right). The patient did not receive any specific cardiac medication or coronary intervention.

BERNWARD LAUER HOLGER THIELE GERHARD SCHULER laub@server3.medizin.uni-leipzig.de
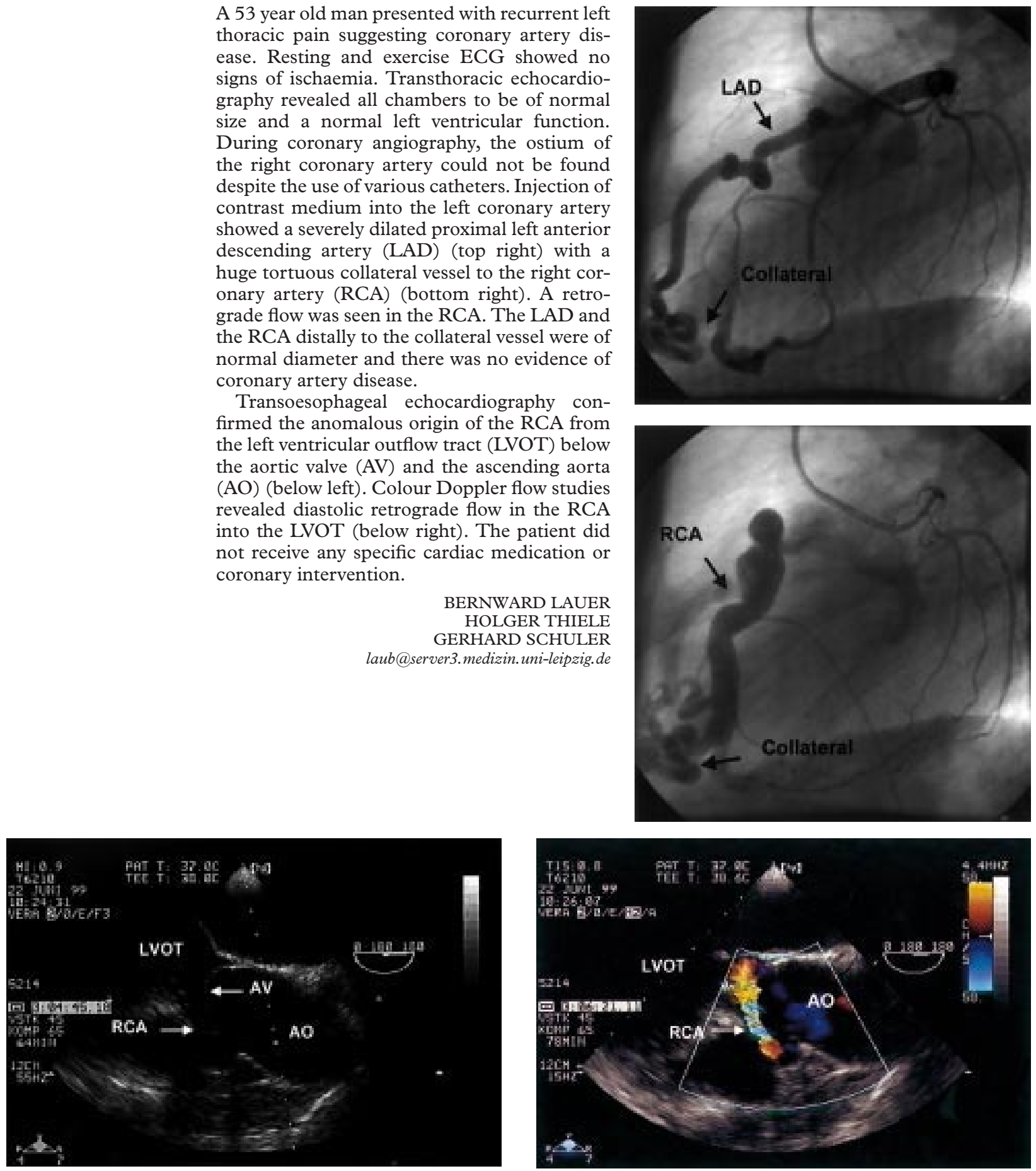